\title{
A low-scatter survey-based mass proxy for clusters of galaxies
}

\author{
S. Andreon
}

INAF - Osservatorio Astronomico di Brera, via Brera 28, 20121 Milano, Italy

e-mail: stefano.andreon@brera.inaf.it

Received 24 August 2012 / Accepted 17 October 2012

\section{ABSTRACT}

\begin{abstract}
Estimates of cosmological parameters using galaxy clusters have the scatter in the observable at a given mass as a fundamental parameter. This work computes the amplitude of the scatter for a newly introduced mass proxy, the product of the cluster total luminosity times the mass-to-light ratio, usually referred as stellar mass. The analysis of 12 galaxy clusters with excellent total masses shows a tight correlation between the stellar mass, or stellar fraction, and total mass within $r_{500}$ with negligible intrinsic scatter: the $90 \%$ upper limit is 0.06 dex, the posterior mean is 0.027 dex. This scatter is similar to the one of best-determined mass proxies, such as $Y_{X}$, i.e. the product of $\mathrm{X}$-ray temperature, and gas mass. The size of the cluster sample used to determine the intrinsic scatter is small, as in previous works proposing low-scatter proxies because very accurate masses are needed to infer very small values of intrinsic scatter. Three-quarters of the studied clusters have $\lg M \lessgtr 14 M_{\odot}$, which is advantageous from a cosmological perspective because these clusters are far more abundant than more massive clusters. At the difference of other mass proxies such as $Y_{\mathrm{X}}$, stellar mass can be determined with survey data up to at least $z=0.9$ using upcoming optical near-infrared surveys, such as DES and Euclid, or even with currently available surveys, covering however smaller solid angles. On the other end, the uncertainty about the predicted mass of a single cluster is large, 0.21 to $0.32 \mathrm{dex}$, depending on cluster richness. This is largely because the proxy itself has $\approx 0.10 \mathrm{dex}$ errors for clusters of $\lg M \lessgtr 14 M_{\odot}$ mass.
\end{abstract}

Key words. galaxies: clusters: general - galaxies: stellar content - galaxies: luminosity function, mass function cosmology: observations - methods: statistical - cosmological parameters

\section{Introduction}

Galaxy clusters are the most massive bound structures. These structures, as well as galaxy groups, in this work are called halos, a term that does not require an arbitrary boundary between the two types of objects. Halos are both cosmological probes and giant physics laboratories. Halos are multicomponent systems formed mostly by dark matter and by baryons in the form of gas and stars. The proportion of gas (intra-cluster medium) and star components is $10: 1$ at $\lg M \sim 14.5 M_{\odot}$, but $1: 1$ at $\lg M \sim 13.5 M_{\odot}$ (e.g. Andreon 2010, and reference therein) and $1: 2$ at $\lg M \sim 12.5-13 M_{\odot}$ (Dai et al. 2010; Humphrey et al. 2011, 2012). The cluster mass function is steep (e.g. Press \& Schecther 1974; Jenkins et al. 2001), and therefore most halos have low masses and thus comparable amounts of stars and gas. Therefore, in all but the shallowest (in mass) surveys, galaxies and gas contain similar amounts of baryons.

The efficient use of clusters as cosmological probes requires a low-scatter mass proxy because cosmological constraints become looser and looser with increasing scatter between mass and mass proxy (e.g. Lima \& Hu 2005). Quantities derived from survey data have generally large scatter: richness and notcore-excised X-ray luminosities have a 0.19 dex intrinsic scatter (richness: Andreon \& Hurn 2010, X-ray luminosity: Vikhlinin et al. 2009a; Mantz et al. 2010a; see Andreon \& Hurn 2010, for a comparison of the predicted mass uncertainties of these two proxies). Instead, the intrinsic scatter of the SZ mass proxy has yet to be robustly measured from the data (Allen et al. 2011). Proxies based on higher quality data perform better: coreexcised X-ray luminosities have a small, and for this reason poorly determined, intrinsic scatter of 0.015-0.025 dex (Mantz et al. 2010a) above $\lg M \sim 14.5 M_{\odot}$ and an unknown scatter below; $Y_{\mathrm{X}}$ shows a similarly small and poorly determined intrinsic scatter of 0.052 dex above $\lg M \sim 14.5 M_{\odot}$ (Mantz et al. 2010a) or 0.07 above $\lg M \sim 14 M_{\odot}$ (Arnaud et al. 2007).

More complex proxies, which combine information from, say, X-ray and SZ data, should perform even better than those using only one of them. In practice, one should have high-quality data for both proxies, not just for one of them. This usually strongly reduces the usable sample and makes it harder to derive the selection function needed to determine of cosmological constraints.

The scatter in observable at a given mass, and not the other way around, is a parameter appearing explicitly in the estimates of cosmological parameters based on galaxy clusters counts and is thus a fundamental parameter. In fact, $\sigma_{\text {proxy } \mid M}$ has been used in past cosmological estimates using cluster counts (Vikhlinin et al. 2009; Mantz et al. 2010b; Rozo et al. 2010). To understand why this scatter, and not the other way around, appears in cosmological estimates one must recall that cosmological parameters are constrained by changing them until the predicted abundance, as a function of the observable, matches the observed distribution. Thus, one needs to go from the mass function, $p(M)$, to the predicted distribution in the observable, $p$ (proxy). Since $p($ proxy $) \propto p($ proxy $\mid M) * p(M)$ (Bayes' theorem, see also Lima \& Hu 2005; and Rozo et al. 2010, for its cosmological application), $p$ (proxy $\mid M)$ (whose minimal parametrisation is given by the scatter at a given mass, $\left.\sigma_{\text {proxy } \mid M}\right)$ is needed. One of the aims of this work is to compute this quantity and compare it to the scatter of other mass proxies, completing the similar exercise done in the review paper of Allen et al. (2011) for other mass proxies (their Sect. 3.3.4)

It is worth emphasizing that the scatter computed the other way around, in mass at a given observable, is also an interesting 
quantity because it is an approximation of how well one may estimate the mass of a single cluster. To be precise, $\sigma_{M \mid \text { proxy }}$ alone is not enough. The whole probability distribution $p(M \mid$ proxy $)$ is needed ${ }^{1}$ because one should also at least account for the proxy uncertainty and for the uncertainties in the mean relation between mass and proxy. The width of the posterior predictive distribution encapsulates all these uncertainties (including less obvious ones, such as errors coming from extrapolations) and has been used in Andreon \& Hurn (2010) to compare the performances of $L_{\mathrm{X}}$ and $n_{200}$ as estimators of the mass of individual clusters.

We emphasise that, once the intrinsic scatter is smaller than the mass error, its value is poorly determined (for all mass proxies) and should be read as an upper limit because of the uncertainty of the mass error and of the likelihood function (often assumed to be Gaussian). Small changes of them imply large changes in the derived intrinsic scatter when the latter is comparable to mass errors. So, although in principle an intrinsic scatter much smaller than the mass error can be determined from data of arbitrary precision, in practice values smaller than mass errors should read as upper limits.

In this paper, we introduce a new mass proxy, the stellar mass, which shows a negligible small intrinsic scatter with hydrostatic mass. Stellar mass is derived as in previous works (e.g. Gonzalez et al. 2007; Andreon 2010): it is given by the stellar $\mathrm{M} / \mathrm{L}$ ratio times the total cluster luminosity. Three-quarters of the studied clusters have $\lg M \lesssim 14 M_{\odot}$, which is advantageous from a cosmological perspective because these clusters are far more abundant than more massive clusters. Furthermore, we show that stellar masses can be derived from imaging data, making it possible to determine the mass proxy up to at least $z=0.9$ with both current and upcoming imaging surveys.

An optical mass proxy with small scatter is of paramount importance, given the current large investment in optical and nearinfrared surveys, such as the Dark Energy Survey (DES ${ }^{2}$, Abbott et al. 2005), Euclid ${ }^{3}$ (Laureijs et al. 2011), and the planned Large Sinoptic Survey Telescope (Ivezic et al. 2008). All these surveys cover thousands of square degrees in several bands with a depth appropriate to derive the proxy value to $z=1$ and beyond (see Sect. 3.3 for details).

Throughout this paper, we assume $\Omega_{\mathrm{M}}=0.3, \Omega_{\Lambda}=0.7$, and $H_{0}=70 \mathrm{~km} \mathrm{~s}^{-1} \mathrm{Mpc}^{-1}$. Magnitudes are quoted in their native system (quasi-AB for Sloan Digital Sky Survey (SDSS) magnitudes). All logarithms in this work are on base ten.

\section{Data and sample}

We started from clusters in Vikhlinin et al. (2006) and Sun et al. (2009) with: a) hydrostatic mass errors lower than $25 \%$ to retain a sample of clusters with accurately measured masses. In practice, because of the other constraints, the typical mass error of halos retained in the sample is $0.05 \mathrm{dex}$; b) inside the SDSS 8th data release (Aihara et al. 2011); c) with redshift between $0.02<z<0.14$, and d) mass larger than $10^{13.2} M_{\odot}$ to keep clusters for which the stellar mass can be accurately measured. In practice, the redshift range of the retained sample, listed in Table 1, is narrower than imposed $(0.02<z<0.078)$

\footnotetext{
1 An appreciation of the difference between $p(x \mid y)$ and $p(y \mid x)$ may, following D'Agostini (2012), be obtained by replacing $x$ with woman and $y$ with senator, obtaining $\approx 10-60 \%$ for the former (in most countries) and $\ll 0.1 \%$ for the latter.

2 http://www. darkenergysurvey.org/

3 http://www. euclid-ec.org
}

Table 1. Stellar masses.

\begin{tabular}{|c|c|c|}
\hline ID & $\begin{array}{c}\log _{10} M_{\star, r_{500}} \\
{\left[M_{\odot}\right]}\end{array}$ & $\begin{array}{c}\log _{10} M_{\star, r_{200}} \\
{\left[M_{\odot}\right]}\end{array}$ \\
\hline Abell 160 & $12.33 \pm 0.12$ & $12.48 \pm 0.10$ \\
\hline Abell 1177 & $12.05 \pm 0.15$ & $12.10 \pm 0.16$ \\
\hline Abell 1795 & $12.66 \pm 0.05$ & $12.80 \pm 0.05$ \\
\hline Abell 1991 & $12.46 \pm 0.12$ & $12.64 \pm 0.09$ \\
\hline Abell 2029 & $12.59 \pm 0.08$ & $12.70 \pm 0.08$ \\
\hline Abell 2092 & $12.34 \pm 0.10$ & $12.44 \pm 0.08$ \\
\hline MKW 4 & $12.25 \pm 0.14$ & $12.40 \pm 0.13$ \\
\hline NGC 4104 & $12.08 \pm 0.20$ & $12.12 \pm 0.25$ \\
\hline NGC 5098 & $12.11 \pm 0.15$ & $12.29 \pm 0.12$ \\
\hline NGC 6269 & $12.39 \pm 0.12$ & $12.58 \pm 0.11$ \\
\hline RX J1022+3830 & $12.43 \pm 0.16$ & $12.51 \pm 0.11$ \\
\hline $3 \mathrm{C} 442 \mathrm{~A}$ & $11.97 \pm 0.14$ & $12.00 \pm 0.14$ \\
\hline Abell 1991 & $12.48 \pm 0.11$ & $12.66 \pm 0.09$ \\
\hline MKW 4 & $12.19 \pm 0.15$ & $12.27 \pm 0.17$ \\
\hline
\end{tabular}

Notes. The last two lines indicate independently derived estimates based on partially independent data.

because of the other constraints. The retained sample consists of 12 unique clusters plus two repeats, i.e. objects listed in both catalogs (MKW4 and Abell 1991) and analysed twice to check analysis-dependent systematics.

The studied sample is based on clusters with available masses, and we have not filtered it by using any optical cluster property, such as the presence of a prominent red sequence or a centrally located brightest cluster galaxy. Therefore, while we are sure that we have not introduced a selection bias, both the starting and the retained samples have an unknown representativity. Nevertheless, the starting sample should not be completely unrepresentative, because it has been successfully used to calibrate the mass- $Y_{\mathrm{X}}$ scaling relation for cosmological estimates (e.g. Vikhlinin et al. 2009).

Measurements are performed within the $r_{500}$ radius ${ }^{4}$, listed in Vikhlinin et al. (2006) and Sun et al. (2009), and within the virial radius, $r_{200}$, derived from $r_{500}$ and $c_{500}$ (also listed in these papers). For three clusters (3C 442, A2092, and NGC 6269), the $r_{200}$ computation assumes a concentration index of four, lacking $c_{500}$ for these two clusters in these papers.

The analysis of the optical data strictly follows Andreon (2010, A10 hereafter). The basic data used in our analysis are $g$ and $r$ photometry from SDSS, down to $r=19$ mag. The latter is the value where the star/galaxy separation becomes uncertain (e.g. Lupton et al. 2002) and is much brighter than the SDSS completeness limit (e.g. Ivezić et al. 2002). Specifically, we use "cmodel" magnitudes for "total" magnitudes and "model" magnitudes for colours. We computed the mass in stars from the cluster total luminosity, the latter computed as the integral of $L$ times the luminosity function of red galaxies. We adopted a Bayesian approach, as done for other clusters (e.g. Andreon 2006, 2010; Andreon et al. 2006, 2008b; Meyers et al. 2012), and we account for the background (galaxies in the cluster line-of-sight), which is estimated outside the cluster turnaround radius. We adopted a Schechter (1976) luminosity function and the likelihood expression given in Andreon et al. (2005), which is an extension of the Sandage et al. (1979) likelihood expression for the case where a background is present. The Bayesian approach allows us to easily propagate uncertainties and their covariance into the

\footnotetext{
$4 r_{\Delta}$ is the radius within which the enclosed average mass density is $\Delta$ times the critical density.
} 
integral of the luminosity function and into the stellar mass. For example, in contrast to all other authors (except Meyers et al. 2012), we do not assume to complete knowledge of the faint end slope of luminosity function and instead marginalise over it, allowing us to propagate this uncertainty in derived quantities. As a visual check, we also computed the luminosity function by binning galaxies in magnitude bins (e.g. Zwicky 1957; Oemler 1974, and many papers since then).

In the luminosity function computation, attention should be paid to saturated stars misclassified as galaxies (we inspected the SDSS images and removed them by hand) and to the brightest cluster galaxy (BCG), which might not be drawn from the Schechter (1976) function (e.g. Tremaine \& Richstone 1977). To deal the latest issue, we fit all galaxies, excluding the BCG, and added its luminosity contribution separately. Finally, we removed by hand very bright (much brighter and larger than the BCG) unrelated galaxies that were spectroscopically in the fore/background of the cluster.

In this paper, we define as red galaxies those within 0.1 redward and 0.2 blueward in $g-r$ of the colour-magnitude relation, as in Andreon \& Hurn (2010) and A10, and in agreement with works mentioned there.

Because of the shallow nature of the SDSS, it misses the flux coming from the galaxy outer regions (e.g. Blanton et al. 2001; Andreon 2002). For this reason we corrected the measured flux by $15 \%$ (Blanton et al. 2001). To compute the luminosity in a sphere from the measured values (derived in a cylinder), we assumed a Navarro et al. (1997) profile. Finally, luminosities are converted in stellar mass by adopting the $M / L$ value derived by Cappellari et al. (2006).

To check whether our stellar mass are over/underestimated, we performed an approximate calculation using simulations, that account only for the effect of the finite sample size of cluster galaxies and of the background subtraction because these two terms were found to be the main sources of error. First, we simulated 1000 clusters, all composed of 73 galaxies whose luminosities are drawn from a Schecther (1976) function with slope $\alpha=-1$. These are best-fit values of NGC 6269. As for real NGC 6269 data, luminosities are drawn down to $M^{*}+5$. To obtain the total luminosity of each simulated cluster, we summed luminosities of the simulated galaxies (Schechter's draws). Now, we needed to simulate the uncertainty associated to the background subtraction. We took a power-law to describe the background counts with best-fit parameters measured for NGC 6269 background area (slope 0.4 and 27 background galaxies per unit cluster solid angle). Next, we added the luminosity of a realization of the background and removed the luminosity of another realization to get the total net cluster luminosity. Since the number of background galaxies is subject to Poisson fluctuations, the number of draws for the two background realizations is allowed to fluctuate Poissonianly. Finally, since the background for NGC 6269 is estimated on a solid angle 12 times larger than the cluster area, our simulation also modelled this. The total net luminosity showed a scatter, from simulation to simulation, of 0.11 dex. For NGC 6269 we derived 0.12 dex from real data (Table 2), the latter also accounting for other (minor) sources of error not modelled in this simulation. The two estimates are close enough not to warrant a more complex simulation and show that our stellar mass errors are accurate.

Several of our steps are only useful for putting the observable, the stellar mass, in standard units and can be safely removed without affecting the quality (intrinsic scatter) of the proposed mass proxy. We can skip the conversion from luminosity to mass because this is a single number and therefore has no effect on the scatter around the mean relation (it only changes the intercept, i.e. the unit of the quantity being measured). In the same vein, we may also remove the correction for the missing flux, which again only affects the intercept because it is a single number. Similarly, the conversion from flux in a cylinder to flux into a sphere is a multiplicative term with a negligible dependency on concentration (it then affects only the intercept). Because of the depth in the cluster rest-frame of the photometry used, the light emitted by $r>19$ mag galaxies is also negligible and could be ignored. In short, stellar mass observationally measures the amount of light emitted from red detected galaxies, and several of our operations are only useful to obtain standard units for this quantity.

As mentioned, these "corrections" affect the absolute value of the intercept of the mass-proxy relation. In other observational conditions (e.g. for shallow observations of distant clusters), these corrections may take different values and differ from cluster to cluster. Therefore, we prefer to characterise the proxy "stellar mass" rather than the observation-dependent raw cluster luminosity, which likely has a larger scatter with mass (for widely varying observational data) and an observationdependent intercept. This choice also simplifies the comparison with recent works.

\section{Results}

Table 1 gives the stellar masses found within $r_{500}$ and $r_{200}$ and their errors. Typically, stellar masses of clusters in our sample have 0.11 dex errors. Two clusters appeared twice in our sample and were independently analysed based on partially independent data (e.g. different background regions, partially different cluster regions because of the numerically different $r_{500}$ values, independent fits, different $c_{500}$ ). We found nearly identical stellar masses showing the negligible effect of non-modelled sources of error. Three clusters (Abell 160, MKW 4, and RX J1022) are in common with A10 and have nearly identical stellar masses within $r_{200}$. A10 used different $r_{200}$ values (derived using a caustic analysis), different background regions and a former release of the SDSS, which implements different types of magnitudes.

In order to fit the trend between stellar and total mass, we use a standard Bayesian fitting model. Essentially, the model assumes that the true stellar mass and true halo mass are linearly related with some intrinsic scatter. However, rather than having these true values, we have noisy measurements of both stellar mass and halo mass, with noise amplitude different from point to point. The code to perform the computation is given in the Appendix A of A10 (ses also Andreon 2006, 2008; Andreon et al. 2006, 2008a,b; Andreon \& Hurn 2010; Kelly 2007).

Using the (fitting) model above, we found for our sample of 12 clusters:

$\lg M_{\star, r_{500}}=(0.38 \pm 0.07)\left(\log M_{500}-14.5\right)+12.53 \pm 0.04$.

Unless otherwise stated, results of the statistical computations are quoted in the form $x \pm y$, where $x$ is the posterior mean and $y$ is the posterior standard deviation.

Figure 1 shows the relation between stellar mass and halo mass, observed data, the mean scaling and its $68 \%$ uncertainty and the mean intrinsic scatter around the mean relation. The intercept and slope marginals are well approximated by Gaussians. The (posterior) probability distribution of the intrinsic scatter $\sigma_{\text {scat }}$ is an exponentially decreasing function (Fig. 2), i.e. the intrinsic scatter is too small to be reliably measured with the data on hand. The same conclusion may also qualitatively inferred 


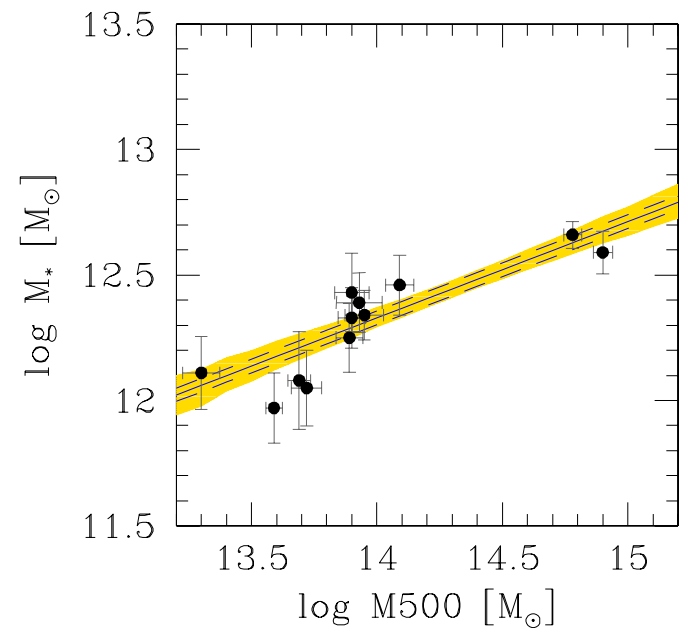

Fig. 1. Stellar vs. total mass. The mean relation between stellar mass and halo mass is marked with a solid line, its $68 \%$ uncertainty is shaded (in yellow). The dashed lines show the mean relation plus or minus the (posterior mean) intrinsic scatter $\sigma_{\text {scat }}$. Error bars on the data points represent observed errors for both variables.

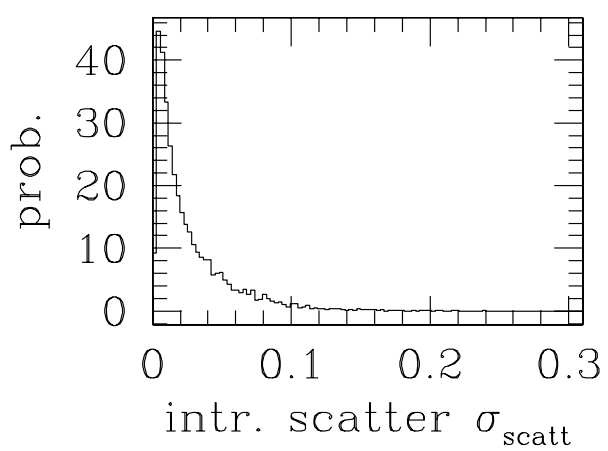

Fig. 2. Posterior probability distribution of the intrinsic scatter $\sigma_{\lg M_{\star} \mid M_{500}}$.

by noting that the mean model crosses the error bars of almost all points. Data are adequately described by the model: we generated 5000 fake data sets from the model, computed their $\chi^{2}$, and found that $71 \%$ of them show a $\chi^{2}$ larger than the (true) data on hand (an extreme $p$-value, say $\lesssim 1 \%$ or $\gtrsim 99 \%$ would call for a model revision). In this $p$-value computation, we accounted for the uncertainty of the regression parameters (intercept, slope, and intrinsic scatter) by adopting Bayesian $p$-values (Gelman et al. 2004; Andreon 2011a).

The $90 \%$ upper limit to the intrinsic stellar mass scatter at a given halo mass, $\sigma_{\text {scat }}=\sigma_{\lg M_{\star} \mid M_{500}}$, is 0.06 dex. The mean and median values are 0.027 and $0.017 \mathrm{dex}$.

As mentioned in Sect. 2, we also measured stellar masses within $r_{200}$. Using the (fitting) model above, we found for our sample of 12 clusters:

$$
\lg M_{\star, r_{200}}=(0.38 \pm 0.07)\left(\log M_{500}-14.5\right)+12.65 \pm 0.05 .
$$

Figure 3 shows the relation between stellar mass and halo mass, observed data, the mean scaling and its $68 \%$ uncertainty and the mean intrinsic scatter around the mean relation for these stellar masses.

As for $r_{500}$, the intercept and slope marginals are well approximated by Gaussians, and the (posterior) probability distribution of the intrinsic scatter $\sigma_{\text {scat }}$ is an exponentially decreasing function with posterior mean $0.05 \pm 0.05$, median 0.03 , and $90 \%$

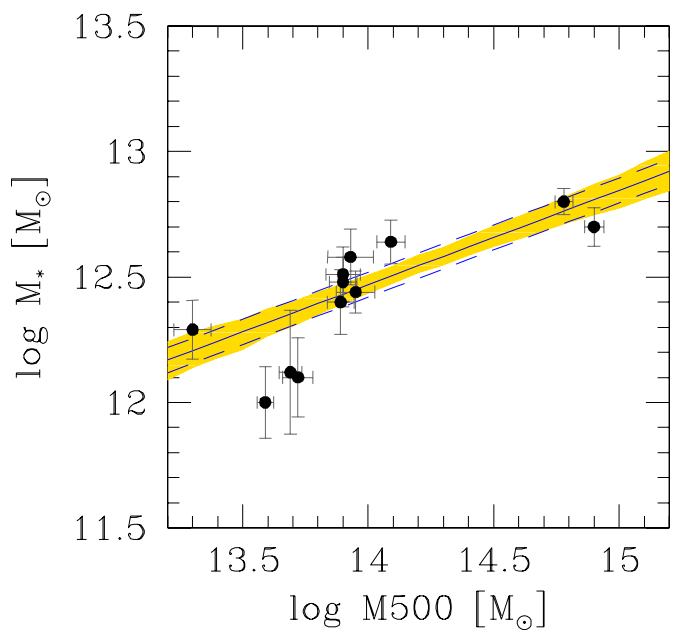

Fig. 3. Stellar vs. total mass. As Fig. 1, but for stellar masses measured within $r_{200}$.

upper limit of 0.12 dex. Also in this case, the data are adequately described by the model (Bayesian $p$-value: $30 \%$ ).

Compared to the relation found using stellar masses measured within the smaller radius $r_{500}$, we found an identical slope, a larger normalization (because stellar masses are integrated in larger apertures), and a somewhat looser constraint on the intrinsic scatter (expected because we are now computing stellar masses in extrapolated $r_{200}$ values). Therefore, the tight scaling does not seem to be a unique feature of the $r_{500}$ radius.

The slope, derived here from hydrostatic masses within $r_{500}$, is in agreement with what was found in A10 for a much larger sample of 52 clusters using dynamical masses within $r_{200}$. The latter sample is not restricted to clusters appearing to be relaxed in X-ray (unlike most samples dealing with $Y_{\mathrm{X}}$ ) and consists of a random sampling of an X-ray flux limited sample (as detailed in A10 and also shown by a numerical simulation in Andreon \& Bergé 2012). A10 uses caustics and velocity dispersion-based masses within $r_{200}$ and found a slope of $0.45 \pm 0.08$ and $0.53 \pm 0.08$ vs. $0.38 \pm 0.07$ found in this work. The agreement of the derived slope between hydrostatic-, caustics-, and velocity dispersion-based masses indicates the absence of a gross tilt between the three mass scales. It also indicates that the current slope is not driven by clusters at the extreme of the mass range. The A10 large sample also offers the opportunity of checking if the found small $\sigma_{\text {scatt }}$ is an unwanted consequence of the small sample size. To this aim, we recomputed the posterior distribution of $\sigma_{\text {scatt }}$ by forcing the slope to be $0.45 \pm 0.08$ (i.e. adopting the latter as prior, in place of the originally adopted Student- $t$ prior) and found an indistinguishable posterior probability distribution. This indicates that the small scatter is not due to overfitting.

The scatter we found in the present work is lower than, although consistent with at $95 \%$, the scatter found in A10 using noisier masses (0.14 vs. 0.05 dex errors for masses within $r_{200}$ ). As mentioned, intrinsic scatter values smaller than the mass errors (like those in A10) should be read as upper limits, hence increasing the agreement. Indeed, A10 assumed Gaussian errors (likelihood function) for mass, whereas the later work by Serra et al. (2011) found errors to be asymmetric (i.e. the likelihood is not Gaussian). If the uncertainty in the likelihood shape and in the error noisiness were accounted for, a larger uncertainty on the intrinsic scatter would have been found by A10, improving the current $(95 \%)$ agreement. 
More in general, imprecise measurements of mass (i.e. with $\gg 0.05$ dex errors) are the probable reason why the tight stellar mass vs. halo mass has not been noted before: imprecise errors not accounted for in the statistical analysis tend to leave a residual scatter of the order of a fraction of the mass error, which the fitting algorithm attributes to the intrinsic scatter term.

\subsection{The usefulness of stellar masses for cluster samples}

How does the scatter in stellar mass at a given halo mass compare to the scatter of other mass proxies? In this comparison, we strictly follow Sect. 3.3.4 of the Allen et al. (2011) review paper.

- the $90 \%$ upper limit of the scatter of $Y_{X}$ at a given mass is 0.06 dex (Mantz et al. 2010a) above $\lg M \sim 14.5 M_{\odot}$ (vs. 0.06 dex for stellar mass within $r_{500}$ ). Other works (Arnaud et al. 2007; Kravtsov et al. 2006) report compatible point estimates, but with unspecified uncertainties;

- the upper limit of Mantz et al. (2010a) to the scatter of the core-excised X-ray luminosity at a given mass is $\$ 0.04$ dex (at an unspecified level) vs. $0.06 \mathrm{dex}$ for stellar mass (90\% upper limit).

To summarise, the stellar mass is a mass proxy with a scatter similar to best-mass proxies. Furthermore, the range in mass in which the relation holds extends to lower masses than other proxies, which is advantageous from a cosmological perspective because clusters of lower mass are far more abundant than more massive clusters. On the other hand, one should not jump to conclusions: the relative merit of different mass proxies cannot be estimated by any single number (e.g. intrinsic scatter). One should also consider the slope of the mass-proxy relation (stellar mass has a shallower slope than core-excised X-ray luminosity and $Y_{\mathrm{X}}$ ) and the size of the sample for which the mass proxy can be measured (stellar mass has probably the largest sample size, being measurable from survey data, see Sect. 3.3), etc. Nevertheless, one of the parameters in cosmological estimates using galaxy cluster counts and using stellar mass as mass proxy is now known and found to be tiny at most.

Our sample size, 12 clusters, is small. However, Vikhlinin et al. (2009b) measured the scatter with only four additional clusters, the widely cited small $Y_{\mathrm{X}}$ scatter of Vikhlinin et al. (2006) and Arnaud et al. (2007) is derived analysing smaller samples, and Kravtsov et al. (2006) proposed the $Y_{\mathrm{X}}$ mass proxy with only four more (simulated) clusters. We have already commented that the scatter remains small even forcing the slope to be the one derived from a much larger cluster sample (with noisier mass errors). Mantz et al. (2010a,b) have a much larger sample, but their $M_{500}$ are obtained via a complex and indirect path passing through $f_{\text {gas }}$ (see their Sect. 2.2.3).

Similarly, concerns that the scatter is found small because of overestimated halo mass errors should be allayed by the fact that: a) there is some overlap among cluster samples; four of our clusters are in common with Vikhlinin et al. (2006), three with Vikhlinin et al. (2009b, they removed MKW4) and that all halo mass errors, for common and uncommon clusters, have been derived by a single team in the same way; and that b) even assuming that mass errors are zero (an unbelievably small value) in order to maximally boost the intrinsic scatter, its posterior is almost unchanged, i.e. stellar mass compares as favorably as the best-mass proxies even in this implausible case. Finally, our numerical simulation in Sect. 2 shows that our stellar mass errors are accurate, and thus we exclude that the small $\sigma_{\text {scatt }}$ are due to inaccurate (overestimated) errors on stellar mass.
Two comments about cluster physics are in order. First, a small scatter between stellar mass in red galaxies and halo mass is expected if during cluster build-up at most a small fraction of galaxies is added to the existing red population, i.e. if the fraction of galaxies that turns from blue to red is small. This is likely the case because the fraction (in number) of blue galaxies is small in clusters at all redshift (Raichoor \& Andreon 2012; Andreon et al. 2006). Moreover, blue galaxies have, on average, lower masses than red ones, and thus their contribution to the total stellar mass is negligible (e.g. Fukugita et al. 1998; Girardi et al. 2000). The found small scatter is instead at variance with numerical simulations (e.g. Young et al. 2011). However, these fail to reproduce, by a large factor, both the stellar fraction and the amount of intracluster light, indicating that probably sub-grid physics is not yet accurately known/implemented.

Second, since the intrinsic scatter of the stellar mass fraction is negligible (all clusters have the same stellar mass at a given cluster mass), the known observed intrinsic scatter in the gas fraction (Sun et al. 2009; A10) cannot be compensated by a correlated scatter in stellar mass fraction to keep constant the total baryon fraction.

\subsection{The usefulness of stellar masses for individual clusters}

In the previous section, we computed the scatter in observable at a given mass, $\sigma_{\text {proxy } \mid M}$. As mentioned in the introduction, cosmological estimates need $p(\operatorname{proxy} \mid M)$, whose minimal parametrisation is given by a Gaussian with $\sigma=\sigma_{\text {proxy|M }}$ (see, e.g. Allen et al. 2011; or Weinberg et al. 2012, reviews). In fact, Vikhlinin et al. (2009), Mantz et al. (2010b), and Rozo et al. (2010) used $\sigma_{\text {proxy } \mid M}$ in their cosmological estimates using cluster counts. For the stellar mass, we found in the previous section a $90 \%$ upper limit of 0.06 dex on $\sigma_{\text {proxy } \mid M}$.

The inverse scatter, $\sigma_{M \mid \text { proxy }}$, is also interesting. As mentioned in the introduction, it is the minimal error of the estimated mass of a given cluster. As detailed in Andreon \& Hurn (2010), the error in the proxy itself, and of course, its availability, limit the usefulness of a given proxy. We computed the inverse scatter as in previous section, fitting the same data set, but using stellar mass as the predictor variable. We found

$\log M_{500}=(2.02 \pm 0.35)\left(\lg M_{\star, r_{500}}-12.5\right)+14.39 \pm 0.09$.

The posterior probability distribution of the intrinsic scatter $\sigma_{\lg M_{500} \mid M_{\star}}$ is an exponentially decreasing function, indicating that the scatter is too small to be determined with the data on hand, with mean 0.08 dex, median 0.04 dex, and $90 \%$ upper limit 0.2 dex. These numbers quantify the uncertainty of the mass of a single cluster predicted from its $\lg M_{\star, r_{500}}$, i.e. its optical luminosity, if all other sources of errors (the above proxy-mass calibration and the proxy uncertainty) are negligible. However, this is unlikely to be the case of stellar masses for clusters with $\lg M \lesssim 14 M_{\odot}$, which are also the majority in our sample. This is because they have a typical 0.1 dex error in stellar mass (Table 1), which propagates into a 0.2 dex error in the predicted mass. The posterior predictive mass uncertainty, which encapsulates both proxy errors and errors coming from the proxy-mass calibration, turns out to be, on average, 0.32 dex for these not massive systems. For our two most massive systems, the predictive mass uncertainty is 0.22 and $0.25 \mathrm{dex}$, whereas for the very rich MS1054 cluster discussed in next section it is $0.21 \mathrm{dex}$.

These uncertainties are larger than those one may obtain from high-quality $\mathrm{X}$-ray-based mass estimates, such as $Y_{\mathrm{X}}$ or core-excised X-ray luminosities. We emphasise, however, that the value of a mass proxy also depends on its availability and 


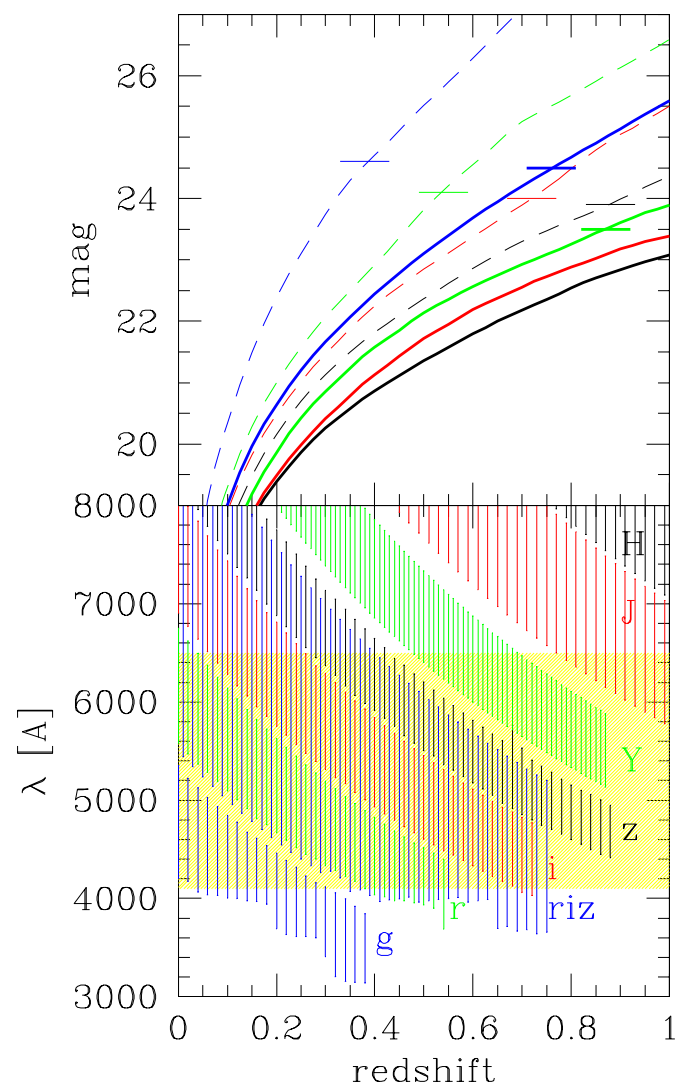

Fig. 4. Depth and wavelength coverage of DES and Euclid surveys. Upper panel: $g, r, i$, and $z$ (from left to right) filters are indicated with thin dashed (blue, green, red and black) lines. riz, $y, J$, and $H$ (from left to right) Eclid filters are indicated with thick solid (blue, green, red, and black) lines. The horizontal tick indicates the $\sim 10 \sigma$ depth. Bottom panel: wavelength coverage of the filters for redshift bins, where galaxies are brighter than the $\sim 10 \sigma$ depth. The shaded (yellow) region marks the wavelength sampling of $g-r$ at $z<0.08$.

that obtaining precise estimates of $Y_{\mathrm{X}}$ or core-excised X-ray luminosities requires expensive, even unfeasible, follow-up observations from space. The value of a mass proxy also depends on how well the mass-proxy relation is calibrated. The $Y_{\mathrm{X}}$ and coreexcised X-ray luminosity vs. mass relations are un-calibrated below $\lg M=14-14.5 M_{\odot}$, the precise value depends on which mass proxy is considered. This is a quite large mass value in the cluster mass function and even more so at intermediate and high redshift. Therefore, stellar masses are a useful mass proxy for clusters of not large mass or when one may not afford the luxury of having the needed high-quality follow-up observations to derive more precise predicted masses.

Strategies to reduce the size of stellar mass errors and thus to increase its quality as mass estimator for individual clusters, are being investigated.

\subsection{On which part of the Universe is stellar mass measurable with current data?}

The analysis of previous sections uses $g-r$ colour and luminosities of galaxies brighter than $r<19$ in the nearby Universe $(z<0.08)$. Figure 4 illustrates how these constraints change with redshifts. The top panel illustrates the apparent luminosity of a red $z=0.08 r=19$ galaxy, modelled as a $z_{\mathrm{f}}=5$ single stellar population using the 2007 version of the Bruzual \& Charlot (2003) synthesis population model, for different filters: $g, r, i$, and $z$ for the Dark Energy Survey (Abbott et al. 2005) and riz, $Y$, $J$, and $H$ for Euclid (Laureijs et al. 2011) with the corresponding $\sim 10 \sigma$ depth (horizontal ticks). The bottom panel illustrates the $\lambda$ range sampled by these filters. Only redshift bins where galaxies are brighter than the $10 \sigma$ depth are plotted. The shaded yellow is the $\lambda$ range sampled by $g-r$ at $z<0.08$. As the figure shows, we always have two filters in the shaded region, i.e. up to $z=0.9$, these data have the depth and wavelength coverage appropriate to measure stellar masses as we did at $z<0.08$. Indeed, an even larger redshift range is accessible for the massive clusters, because the depth requirement can be safely relaxed, as it is not necessary to accurately measure stellar masses for these objects. We further emphasise that these depths, which cover several thousands of $\mathrm{deg}^{2}$, are quite comparable to the depths of surveys available right now covering a ten of $\mathrm{deg}^{2}$, such as the deep fields of the CFHTLS (Cuillandre \& Bertin 2006), VISTAVIDEO $^{5}$ (Jarvis et al. 2012), or WIRDS (Bielby et al. 2012). Repeating this exercise with the planned Large Sinoptic Survey Telescope $10 \mathrm{yr}$ integration (Ivezic et al. 2008), we found that $z=1.2$ may be easily reached.

In order to use stellar masses for large cluster samples, the integral of the luminosity function computation should not include any manual step or complementary (e.g. spectroscopic) data. In our analysis of nearby clusters, we removed by hand saturated stars misclassified as galaxies and used spectroscopy to identify foreground galaxies brighter and larger than the BCG. The first step can be automatically implemented by using the central object intensity (or any flag based on it). The second step may use galaxy sizes and photometric redshifts in place of spectroscopic redshifts that are perhaps not available. Therefore, automatic computation is possible for large cluster samples.

In order to test whether the increased galaxy background at high redshift may be detrimental for an accurate measurement of the stellar mass, we computed the stellar mass of two high redshift clusters using real data.

We first consider MS1054 (Gioia et al. 1990) at $z=0.83$, a massive cluster of galaxies $\left(\lg M_{500} \approx 14.8 M_{\odot}\right.$, Jee et al. 2005) for which we consider only $V<24.7 \mathrm{mag}$ (to match depths) and $V-I$ colour, coming from FIRES (Labbe et al. 2003; Förster Schreiber et al. 2006), which was already used in Andreon (2006) to measure the luminosity function of its red galaxies (see his Fig. 4). Figure 5 shows the $V-I$ vs. $I$ diagram of galaxies in the cluster line-of-sight (top panel) and in a reference line-of-sight (bottom panel). As is fairly obvious, the background contribution, depicted in the bottom panel, at the colour of red galaxies (inside the dashed lines), is negligible. Our luminosity function determination of red galaxies of several clusters (e.g. of the 28 clusters in Andreon 2008, up to $z=1.3$ ) observationally confirms that a low background can be easily achieved by adopting a colour index bracketing the $4000 \AA$ break and that the background is not a large penalty for red galaxies up to the highest redshifts. Furthermore, the background contamination can be further reduced using photometric redshift. This situation extends up to the largest redshifts: Andreon (2011b) found that there are on average 3.6 back/foreground red galaxies in the $z \sim 2.2$ JKCS041 cluster (Andreon et al. 2009; Andreon $\&$ Huertas-Company 2011) line-of-sight from measurements all around the cluster vs. 23 galaxies on the red sequence. We computed the MS1054 stellar mass as we did for lower redshift clusters and found an error of 0.04 dex. The error is small because of

\footnotetext{
5 http://star-www.herts.ac.uk/\%7Emjarvis/video/index. html
} 


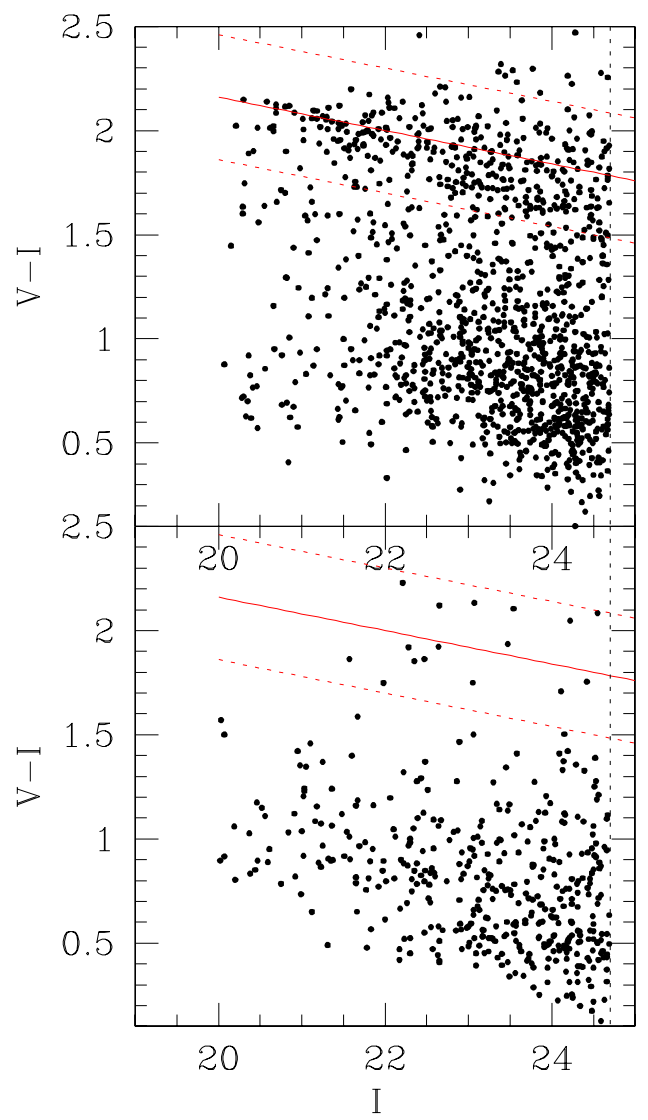

Fig. 5. Colour-magnitude relation in the MS1054 cluster (top panel) and control field (bottom panel) line-of-sight directions. The solid line marks the colour-magnitude relation, whereas the dotted lines delimit the portion of plane that qualifies galaxies to be called red. The vertical line marks the considered limiting depth. In this figure, we use magnitudes in the Vega system, for comparison with the similar figures in Andreon (2006).

the large richness of MS1054 and the low background contamination at the red sequence colour.

As a worse case scenario, we consider RzCS052 at $z=1.016$ (Andreon et al. 2008a,b). The cluster is still a massive one $\left(\lg M_{500} \approx 14.4 M_{\odot}\right.$, Andreon et al. 2008a), barely detected in $\sim 10 \mathrm{ks}$ XMM observations ${ }^{6}$. The cluster is at a redshift larger than the maximal one that we conservatively said possible for stellar mass computation, and even more so with the available data (from Andreon et al. 2008b), which are shallower (by a few tenths of mag) than the DES+Euclid surveys, and made the derivation of stellar mass challenging. The colour-magnitude plot and luminosity function of the red galaxies of RzCS052 are depicted in Figs. 2 and 4 of Andreon et al. (2008b). We computed the stellar mass as we did for lower redshift clusters and found an error of 0.1 dex, in spite of having taken a cluster outside the redshift range claimed to be accessible, having used data of lower depth than upcoming surveys, and having discarded the multicolour data available for this cluster (and for the considered surveys).

As for other mass proxies (e.g. $Y_{\mathrm{X}}$ and $L_{\mathrm{X}}$ ), the quoted error assumes to perfectly know the evolution of the intercept of the relation between mass and mass proxy. In the case of stellar masses, the evolution is modulated by the $\mathrm{M} / \mathrm{L}$ ratio of red

\footnotetext{
6 There is a typo into the exponent of the cluster flux reported in Andreon et al. 2008a: the correct flux is $1.2 \times 10^{-14} \mathrm{erg}^{-1} \mathrm{~s}^{-1} \mathrm{~cm}^{-2}$, as one may discern from the quoted $L_{\mathrm{X}}$.
}

galaxies, which is robustly known (e.g. Treu et al. 2005; Holden et al. 2010) up to at least $z \sim 1$. Instead, the evolution of other mass proxies is poorly known (e.g. the evolution of $Y_{\mathrm{X}}$ or $L_{\mathrm{X}}$ with $z$, Andreon et al. 2011).

\section{Summary}

The analysis of a sample of clusters of the nearby Universe $(z<0.08)$, which is of low cardinality because of the need of high-quality mass estimates ( 0.05 dex mass errors), shows that stellar mass, derived measuring the luminosity emitted by red galaxies only, has a tiny scatter with halo mass, similar to bestmass proxies. We show that stellar masses can be measured with survey-quality data (optical and near-infrared imaging) typical of current (on some square degrees) and upcoming (on several thousand of square degrees) surveys up to $z=0.9-1.2$.

Three-quarters of the studied clusters have $\lg M \lesssim 14 M_{\odot}$, which is advantageous from a cosmological perspective because these clusters are far more abundant than more massive clusters. In constrast to other mass proxies, we have robust knowledge about the evolution of the stellar mass proxy at a given halo mass. This is because the mass evolution is modulated by the evolution of the $\mathrm{M} / \mathrm{L}$ of red galaxies, which is observationally well constrained up to at least $z \sim 1$. We emphasise, however, that a small scatter is an essential property for a good mass proxy, but this number alone cannot completely characterise a mass proxy: availability of the mass proxy and its error also are two essential properties. We also emphasise that if the purpose of a mass proxy is to derive the mass of an individual cluster (as opposed to a sample), survey-based stellar masses should be viewed as a complementary mass proxy to be used when one may not afford the luxury of having non-survey high-quality masses requested for better mass proxies, such as $Y_{\mathrm{X}}$ or coreexcised luminosities, or when these measurements are infeasible or uncalibrated.

Clusters in our sample display an intrinsic scatter in gas fraction (Sun et al. 2009; Andreon 2010) and a tiny scatter in stellar fraction. Therefore, the latter cannot compensate for the scatter of the former to keep the total baryon fraction constant.

Acknowledgements. I thank Antonaldo Diaferio, Fabio Gastaldello and Federico Marulli for useful discussions. I warmly thank Ben Maughan for useful suggestions that helped to improve the quality of my paper. For the standard SDSS, NED, and DSS acknowledgements see: http://www.sdss3.org/, http://nedwww.ipac.caltech.edu/, and http://archive.stsci.edu/ dss/acknowledging.html.

\section{References}

Abbott, T., Aldering, G., Annis, J., et al. 2005 [arXiv: astro-ph/0510346]

Aihara, H., Allende Prieto, C., An, D., et al. 2011, ApJS, 193, 29

Allen, S. W., Evrard, A. E., \& Mantz, A. B. 2011, ARA\&A, 49, 409

Andreon, S. 2002, A\&A, 382, 495

Andreon, S. 2006, MNRAS, 369, 969

Andreon S., 2008, MNRAS, 386, 1045

Andreon, S. 2010, MNRAS, 407, 263 (A10)

Andreon, S. 2011a, in Astrostatistical Challenges for the New Astronomy, ed. J.

Hilbe, Springer Series on Astrostatistics [arXiv: 1112.3652]

Andreon, S. 2011b, A\&A, 529, L5

Andreon, S., \& Bergé, J. 2012, A\&A, 547, A117

Andreon, S., \& Huertas-Company, M. 2011, A\&A, 526, A11

Andreon, S., \& Hurn, M. A. 2010, MNRAS, 404, 1922

Andreon, S., Punzi, G., \& Grado, A. 2005, MNRAS, 360, 727

Andreon, S., Cuillandre, J.-C., Puddu, E., \& Mellier, Y. 2006a, MNRAS, 372, 60

Andreon, S., Quintana, H., Tajer, M., Galaz, G., \& Surdej, J. 2006b, MNRAS, 365,915

Andreon, S., De Propris, R., Puddu, E., Giordano, L., \& Quintana, H. 2008a, MNRAS, 383, 102 
Andreon, S., Puddu, E., de Propris, R., \& Cuillandre, J.-C. 2008b, MNRAS, 385, 979

Andreon, S., Maughan, B., Trinchieri, G., \& Kurk, J. 2009, A\&A, 507, 147

Andreon, S., Trinchieri, G., \& Pizzolato, F. 2011, MNRAS, 412, 2391

Arnaud, M., Pointecouteau, E., \& Pratt, G. W. 2007, A\&A, 474, L37

Bielby, R., Hudelot, P., McCracken, H. J., et al. 2012, A\&A, 545, A23

Blanton, M. R., Dalcanton, J., Eisenstein, D., et al. 2001, AJ, 121, 2358

Bruzual, G., \& Charlot, S. 2003, MNRAS, 344, 1000

Cappellari, M., Bacon, R., Bureau, M., et al. 2006, MNRAS, 366, 1126

Cuillandre, J.-C., \& Bertin, E. 2006, SF2A-2006: Semaine de l'Astrophysique Francaise, 265

Dai, X., Bregman, J. N., Kochanek, C. S., \& Rasia, E. 2010, ApJ, 719, 119

D'Agostini, G. 2011, unpublished [arXiv: 1112 . 3620]

Förster Schreiber, N. M., Franx, M., Labbé, I., et al. 2006, AJ, 131, 1891

Fukugita, M., Hogan, C. J., \& Peebles, P. J. E. 1998, ApJ, 503, 518

Gelman, A., Carlin, J., Stern, H., \& Rubin, D. 2004, Bayesian Data Analysis (Chapman \& Hall/CRC)

Gioia, I. M., Maccacaro, T., Schild, R. E., et al. 1990, ApJS, 72, 567

Girardi, M., Borgani, S., Giuricin, G., Mardirossian, F., \& Mezzetti, M. 2000, ApJ, 530, 62

Gonzalez, A. H., Zaritsky, D., \& Zabludoff, A. I. 2007, ApJ, 666, 147

Holden, B. P., van der Wel, A., Kelson, D. D., Franx, M., \& Illingworth, G. D. 2010, ApJ, 724, 714

Humphrey, P. J., Buote, D. A., Canizares, C. R., Fabian, A. C., \& Miller, J. M. 2011, ApJ, 729, 53

Humphrey, P. J., Buote, D. A., O’Sullivan, E., \& Ponman, T. J. 2012, ApJ, 755, 166

Ivezić, Ž., Menou, K., Knapp, G. R., et al. 2002, AJ, 124, 2364

Ivezic, Z., Tyson, J. A., Acosta, E., et al. 2008, unpublished [arXiv: 0805 . 2366]

Jarvis, M. J., Bonfield, D. G., Bruce, V. A., et al. 2012, MNRAS, in press [arXiv: 1206.4263]

Jee, M. J., White, R. L., Ford, H. C., et al. 2005, ApJ, 634, 813
Jenkins, A., Frenk, C. S., White, S. D. M., et al. 2001, MNRAS, 321, 372 Kelly, B. C. 2007, ApJ, 665, 1489

Kravtsov, A. V., Vikhlinin, A., \& Nagai, D. 2006, ApJ, 650, 128

Labbé, I., Franx, M., Rudnick, G., et al. 2003, AJ, 125, 1107

Laureijs, R., Amiaux, J., Arduini, S., et al. 2011 [arXiv: 1110.3193]

Lima, M., \& Hu, W. 2005, Phys. Rev. D, 72, 043006

Lupton, R. H., Ivezic, Z., Gunn, J. E., et al. 2002, Proc. SPIE, 4836, 350

Mantz, A., Allen, S. W., Ebeling, H., Rapetti, D., \& Drlica-Wagner, A. 2010a, MNRAS, 406, 1773

Mantz, A., Allen, S. W., Rapetti, D., \& Ebeling, H. 2010b, MNRAS, 406, 1759

Meyers, J., Aldering, G., Barbary, K., et al. 2012, ApJ, 750, 1

Navarro, J. F., Frenk, C. S., \& White, S. D. M. 1997, ApJ, 490, 493

Oemler, A. J. 1974, ApJ, 194, 1

Press, W. H., \& Schechter, P. 1974, ApJ, 187, 425

Randall, S. W., Jones, C., Markevitch, M., et al. 2009, ApJ, 700, 1404

Raichoor, A., \& Andreon, S. 2012, A\&A, 543, A19

Rozo, E., Wechsler, R. H., Rykoff, E. S., et al. 2010, ApJ, 708, 645

Sandage, A., Tammann, G. A., \& Yahil, A. 1979, ApJ, 232, 352

Schechter, P. 1976, ApJ, 203, 297

Serra, A. L., Diaferio, A., Murante, G., \& Borgani, S. 2011, MNRAS, 412, 800

Sun, M., Voit, G. M., Donahue, M., et al. 2009, ApJ, 693, 1142

Tremaine, S. D., \& Richstone, D. O. 1977, ApJ, 212, 311

Treu, T., Ellis, R. S., Liao, T. X., \& van Dokkum, P. G. 2005, ApJ, 622, L5

Vikhlinin, A., Kravtsov, A., Forman, W., et al. 2006, ApJ, 640, 691

Vikhlinin, A., Burenin, R. A., Ebeling, H., et al. 2009a, ApJ, 692, 1033

Vikhlinin, A., Kravtsov, A. V., Burenin, R. A., et al. 2009b, ApJ, 692, 1060

Young, O. E., Thomas, P. A., Short, C. J., \& Pearce, F. 2011, MNRAS, 413, 691

Weinberg, D. H., Mortonson, M. J., Eisenstein, D. J., et al. 2012, Phys. Rep. [arXiv: 1201.2434]

Zwicky, F. 1957, Morphological astronomy (Berlin: Springer) 